\title{
ATRIBUTOS FÍSICOS E MATÉRIA ORGÂNICA DE ORGANOSSOLOS HÁPLICOS EM DISTINTOS AMBIENTES NO $\operatorname{BRASIL}^{(1)}$
}

\author{
Adierson Gilvani Ebeling ${ }^{(2)}$, Lucia Helena Cunha dos Anjos ${ }^{(3)}$, Daniel Vidal Pérez ${ }^{(4)}$, \\ Marcos Gervasio Pereira ${ }^{(3)}$ \& Etelvino Henrique Novotny ${ }^{(4)}$
}

\begin{abstract}
RESUMO
Os Organossolos são formados em condições geomórficas e climáticas que favorecem o acúmulo de material orgânico e sua drenagem para uso agrícola conduz a subsidência e modificações no teor de matéria orgânica do solo (MOS), com implicações na sustentabilidade desses solos. $O$ objetivo deste trabalho foi avaliar alguns atributos físicos e sua relação com o teor e grau de decomposição da matéria orgânica de Organossolos Háplicos formados em ambientes hidromórficos, nos Estados do Rio de Janeiro (clima tropical atlântico Aw), Maranhão (tropical Awh) e Paraná (subtropical Cfb), com distintos usos. Foram utilizados os métodos recomendados no Sistema Brasileiro de Classificação de Solos (SiBCS) para caracterização de oito perfis, totalizando 30 horizontes, de Organossolos, sendo o teor de matéria orgânica quantificado pelo método da mufla. Os atributos físicos densidade do solo (Ds), densidade da matéria orgânica (DMO), percentual de material mineral (MM) e resíduo mínimo (RM) refletiram no teor e grau de transformação do material orgânico. A Ds variou de 0,09 a $0,93 \mathrm{Mg} \mathrm{m}^{-3}$, com média de $0,44 \mathrm{Mg} \mathrm{m}^{-3}$ e a DMO, de 0,02 a $0,66 \mathrm{Mg} \mathrm{m}^{-3}$, sendo a média de $0,28 \mathrm{Mg} \mathrm{m}^{-3}$. Observou-se relação diretamente proporcional entre a Ds e os atributos MM e RM. Quanto ao grau de decomposição, os materiais orgânicos foram identificados principalmente nas classes de hêmicos e sápricos; porém, como classes distintas foram obtidas em função do método usado, recomenda-se o uso do índice de pirofosfato e teor de fibras esfregadas em conjunto, definindo as classes por faixa de valores. Os Organossolos com menor alteração antrópica, no Estado do Rio de Janeiro (RJ02 e RJ08), apresentaram os
\end{abstract}

(1) Parte da Tese de Doutorado do primeiro autor apresentada ao Curso de Pós-Graduação em Agronomia - Ciência do Solo (CPGA-CS) da Universidade Federal Rural do Rio de Janeiro - UFRRJ, Seropédica (RJ). Projeto financiado pelo CNPq. Recebido para publicação em 19 de julho de 2012 e aprovado em 12 de abril de 2013.

(2) Pesquisador do Centro Estadual de Pesquisa em Desenvolvimento Rural Sustentável, Pesagro/Rio. Estrada Velha de Glicério, km 03, Horto. Caixa Postal 119.371. CEP 27910-970 Macaé (RJ). E-mail: adiersonge@gmail.com

(3) Professor Associado IV, Departamento de Solos, Instituto de Agronomia, UFRRJ. Bolsistas de Produtividade do CNPq e da FAPERJ. BR 465, km 7. CEP 23890-000 Seropédica (RJ). E-mail: lanjos@ufrrj.br; gervasio@ufrrj.br

(4) Pesquisador da Embrapa Solos. Bolsista Produtividade do CNPq e FAPERJ. Rua Jardim Botânico, 1024. CEP 22460-000 Rio de Janeiro (RJ). E-mail: daniel.perez@embrapa.br; etelvino.novotny@embrapa.br 
maiores teores de matéria orgânica e os menores valores de Ds, DMO e MM. Assim, esses atributos são relevantes como indicadores do potencial de subsidência e da degradação dos Organossolos, solos relevantes pelo seu alto estoque de carbono e para a preservação de aquíferos.

Termos de indexação: solos orgânicos, grau de decomposição da matéria orgânica, subsidência.

\title{
SUMMARY: PHYSICAL PROPERTIES AND ORGANIC MATTER OF HISTOSOLS FROM DIFFERENT ENVIRONMENTS OF BRAZIL
}

\begin{abstract}
Histosols are formed under geomorphic and climate conditions that allow the accumulation of organic material, and their drainage for agriculture leads to the subsidence process and changes in the content of soil organic matter (SOM), with implications in the sustainability of these soils. The objective of this study was to evaluate some physical properties of Histosols and their relationship to SOM content and degree of decomposition in soils formed under hydromorphic conditions in the states of Rio de Janeiro (Aw tropical Atlantic), Maranhão (Awh tropical), and Parana (Cfb subtropical), with different usages. The methods recommended by the Brazilian Soil Classification System (SiBCS) were applied for characterization of the eight profiles, totaling 30 horizons; the organic matter content was quantified using a muffle furnace. The physical properties, soil bulk density (BD), organic matter density (OMD), percentage of mineral material (MM), and minimum residue (MR), reflected the content and transformation degree of the organic matter. The BD values varied from 0.09 to $0.93 \mathrm{Mg} \mathrm{m}^{-3}$, with an average of $0.44 \mathrm{Mg} \mathrm{m}^{-3}$ and $\mathrm{OMD}$ varied from 0.02 to $0.66 \mathrm{Mg} \mathrm{m}^{-3}$, with an average of $0.28 \mathrm{Mg} \mathrm{m}^{-3}$. A directly proportional relationship between $B D$ and the properties $M M$ and $M R$ was observed. In the classification of the decomposition degree, the organic materials were identified mainly as hemic and sapric. Since different classes were obtained according to the method applied, it is recommended the combined usage of the pyrophosphate index and the content of rubbed fibers, defining the classes according to a range of values. In the Histosols with less anthropic alteration, in the State of Rio de Janeiro (profiles RJ02 and RJ08), the levels of organic matter were highest and the values of $B D, O M D$, and MM lowest. Thus, these properties are relevant to indicate the potential of subsidence and degradation in Histosols, an important soil class with regard to carbon storage and preservation of aquifers.
\end{abstract}

Index terms: organic soils, decomposition of organic matter, subsidence.

\section{INTRODUÇÃO}

Os Organossolos no Brasil são utilizados intensamente pela agricultura familiar e têm grande importância ambiental pelo seu elevado teor de matéria orgânica no solo (MOS). Trabalhos como os de Sakai \& Lepsch (1987), Lepsch et al. (1990), Silva et al. (2009) são pontuais e caracterizam solos com altos teores de MOS em uma só paisagem, sendo exceções os trabalhos de Galvão \& Vahl (1996), que estudaram Organossolos do litoral da região Sul do País, e Valladares et al. (2008), que compilaram dados da ordem dos Organossolos em âmbito nacional.

O material parental, nos Organossolos é constituído por deposições de resíduos vegetais em diferentes estádios de decomposição, fragmentos de carvão finamente divididos, substâncias húmicas, biomassa meso e microbiana e outros compostos orgânicos naturalmente presentes no solo, que podem estar associados ao material mineral em proporções variáveis. A deposição e acumulação de matéria orgânica ocorrem em condições de excesso de água (horizonte $\mathrm{H}$ ) ou de drenagem livre (horizonte $\mathrm{O}$ ); nesse último caso, em geral, em ambiente de clima úmido e vegetação altomontana (Santos et al., 2006).

A decomposição, biológica/natural ou antrópica, da MOS nos Organossolos reduz o volume inicial de solo, levando ao processo de subsidência; porém, estruturas mais estáveis e materiais mais humificados podem ser formados, contribuindo para a redução da intensidade do processo, apesar das perdas iniciais (Lützow et al., 2006). As características sápricas, hêmicas ou fíbricas resultam de diferentes taxas de decomposição da MOS em ambientes que aceleram ou retardam a subsidência (Gesch et. al., 2007). Nas áreas agrícolas, as taxas de subsidência estão relacionadas à profundidade dos canais de drenagem, aos diferentes tipos de uso das terras drenadas e à variação do nível do lençol freático ao longo do ano, com consequências inclusive na variação das emissões de $\mathrm{CO}_{2}$ (Pumpanen et al., 2004).

Para avaliar o potencial máximo de subsidência em solo de natureza orgânica, Lynn et al. (1974) 
introduziram a variável denominada resíduo mínimo (RM). O RM se refere a uma espessura remanescente de solo por unidade da medida, após atingir a subsidência máxima. No decorrer do processo de subsidência, além da diminuição do RM (Conceição et al., 1999b), surgem alterações na densidade do solo (Ds) e na porosidade do solo e esses atributos estão diretamente relacionados ao RM. Nos Organossolos, os valores de Ds tendem a ser inferiores a uma unidade e, em alguns componentes dessa ordem, valores inferiores a $0,15 \mathrm{Mg} \mathrm{m}^{-3}$ podem ser atingidos (Kämpf \& Schneider, 1989; Elder \& Lal, 2008; Valladares et al., 2008). Nos Organossolos, a Ds é facilmente alterada pela compactação, seja por máquinas ou pisoteio animal, e pela subsidência e transformação da MOS, após a drenagem. Já, a porosidade do solo depende da natureza do material orgânico, onde os materiais orgânicos do tipo fíbrico tendem a apresentar predomínio de macroporos, enquanto os materiais sápricos, de microporos. Quando drenado e seco, esse material pode endurecer irreversivelmente. Porém, no processo de decomposição e mineralização da MOS, esses macroporos colapsam, diminuindo o tamanho dos poros e também a quantidade de porosidade total (Elder \& Lal, 2008).

Os objetivos deste trabalho foram avaliar os atributos físicos: densidade do solo, densidade da matéria orgânica, resíduo mínimo e teor de material mineral e relacionar esses atributos com o teor e grau de decomposição da matéria orgânica de Organossolos Háplicos, formados em ambientes hidromórficos, em diferentes condições climáticas e sob distintos tipos de uso, nos Estados do Rio de Janeiro, Maranhão e Paraná.

\section{MATERIAL E MÉTODOS}

\section{Localização, características gerais, descrição e coleta dos Organossolos}

Os locais foram selecionados em razão do grau de subsidência, drenagem natural e, ou, artificial das áreas, tipo de uso e cobertura, clima e relevo (Quadro 1). Os perfis foram coletados nos Estados do Rio de Janeiro (municípios de Silva Jardim e Macaé), Maranhão (município de Monção) e Paraná (município de Ponta Grossa), em clima quente e úmido e ambiente de bacia (RJ e MA) e em clima frio e planalto (PR). Dos oito perfis coletados, seis se encontravam em várzea com lençol freático elevado e muito pouca influência marinha e dois foram coletados em áreas que foram drenadas e o lençol freático não mais atinge a parte superior do perfil.

Os perfis foram descritos e coletados de acordo com Santos et al. (2005) e classificados, segundo o Sistema Brasileiro de Classificação de Solo (SiBCS) (Santos et al., 2006). Em cada horizonte, foram coletadas amostras de solo deformadas e indeformadas. Essas amostras foram parcialmente secadas ao ar, para evitar o ressecamento total do material, depois destorroadas e passadas por peneira com malha de $2,0 \mathrm{~mm}$, para obter terra fina seca ao ar (TFSA). Amostras de cada horizonte foram armazenadas in natura para as análises do teor de fibras.

\section{Caracterização analítica dos Organossolos}

Os materiais de solo foram caracterizados quanto às propriedades físicas, segundo o SiBCS (Santos et al., 2006). Testes para distinção da natureza do material orgânico e seu grau de decomposição e a proporção de material mineral foram utilizados para a caracterização e classificação do material orgânico, segundo Lynn et al. (1974) e Carter (1993).

A densidade da matéria orgânica (DMO) foi calculada segundo a equação:

$$
\mathrm{DMO}=\mathrm{Ds}-[\mathrm{Ds} \mathrm{x}(\% \text { massa MM/100) }]
$$

em que MM é o material mineral e Ds, a densidade do solo.

O resíduo mínimo (RM) é a estimativa da proporção entre a espessura residual e a original dos horizontes hísticos, alterada por perdas do material orgânico, se o solo for submetido à intensa subsidência (Lynn et al., 1974). O RM é obtido a partir da equação:

$$
\mathrm{RM}=(\mathrm{Ds}-\mathrm{DMO}) / 1,5
$$

em que 1,5 é um valor médio de Ds, atribuído para subsidência total.

$\mathrm{O}$ teor de material mineral (MM) foi determinado pela combustão em mufla:

$$
\begin{aligned}
& \mathrm{MM}=\left(\text { massa seca a } 400{ }^{\circ} \mathrm{C} \text { por } 24 \mathrm{~h}\right) / \\
& \left(\text { massa seca a } 105^{\circ} \mathrm{C} \text { por } 24 \mathrm{~h}\right) \times 100
\end{aligned}
$$

O grau de decomposição do material orgânico foi determinado pelo método de Von Post (Von Post, 1922) e pelo método volumétrico, que separa o material fíbrico do não fíbrico por peneiramento, em que os teores de fibras esfregadas e não esfregadas são expressos em porcentagem, em relação ao material total (fibras e solo). Nesse método, materiais com $<17 \%$ de fibras esfregadas são identificados como sápricos; com valores entre 17 e $40 \%$ de fibras, como hêmicos; e superiores a $40 \%$ de fibras, como fíbricos.

O índice em pirofosfato de sódio (IP) foi obtido por método colorimétrico (Lynn et al., 1974). Uma amostra de solo de estrutura indeformada $\left(2,5 \mathrm{~cm}^{3}\right)$ foi mantida em contato com solução de pirofosfato de sódio por uma noite (aproximadamente 12 h). Após esse tempo, a amostra foi esfregada em papel cromatográfico e a cor comparada com os padrões na página 10YR da carta de cores de solo de Munsell. O índice de pirofosfato (IP) foi calculado subtraindo-se o croma do valor. Valores de IP $>5$ indicam material orgânico fíbrico e valores de IP $\leq 3$ evidenciam material sáprico. 
Quadro 1. Clima, paisagem, vegetação, drenagem, coordenadas, classificação e altitude dos perfis de Organossolos estudados

\begin{tabular}{|c|c|c|c|c|c|c|}
\hline Perfil & Clima & Paisagem & $\begin{array}{l}\text { Vegetação, } \\
\text { drenagem }\end{array}$ & Coordenadas & Classificação & Altitude \\
\hline & & & & & & $\mathrm{m}$ \\
\hline RJ01 & $\begin{array}{l}\text { Tropical } \\
\text { úmido }\end{array}$ & $\begin{array}{l}\text { Várzeas litorâneas } \\
\text { e Mata Atlântica }\end{array}$ & $\begin{array}{l}\text { Floresta higrófil } \\
\text { de várzea e } \\
\text { campo higrófilo - } \\
\text { muito mal drenado }\end{array}$ & $\begin{array}{l}22^{\circ} 35^{\prime} 04.8^{\prime \prime} \mathrm{S} \\
42^{\circ} 15^{\prime} 36.3^{\prime \prime} \mathrm{W}\end{array}$ & $\begin{array}{l}\text { Organossolo } \\
\text { Háplico hêmico } \\
\text { térrico }\end{array}$ & 20 \\
\hline RJ02 & $\begin{array}{l}\text { Tropical } \\
\text { úmido }\end{array}$ & $\begin{array}{l}\text { Várzeas litorâneas } \\
\text { e Mata Atlântica }\end{array}$ & $\begin{array}{l}\text { Floresta higrófila } \\
\text { de várzea - } \\
\text { muito mal drenado }\end{array}$ & $\begin{array}{l}22^{\circ} 31^{\prime} 30.7^{\prime \prime} \mathrm{S} \\
42^{\circ} 20^{\prime} 35.7^{\prime \prime} \mathrm{W}\end{array}$ & $\begin{array}{l}\text { Organossolo } \\
\text { Háplico sáprico } \\
\text { típico }\end{array}$ & 7 \\
\hline RJ03 & $\begin{array}{l}\text { Tropical } \\
\text { úmido }\end{array}$ & $\begin{array}{l}\text { Várzeas litorâneas } \\
\text { e Mata Atlântica }\end{array}$ & $\begin{array}{l}\text { Floresta higrófila } \\
\text { de várzea e campo } \\
\text { higrófilo - mal } \\
\text { drenado }\end{array}$ & $\begin{array}{l}22^{\circ} 17^{\prime} 14.7^{\prime \prime} \mathrm{S} \\
41^{\circ} 52^{\prime} 59.4^{\prime \prime} \mathrm{W}\end{array}$ & $\begin{array}{l}\text { Organossolo } \\
\text { Háplico hêmico } \\
\text { típico }\end{array}$ & 10 \\
\hline RJ04 & $\begin{array}{l}\text { Tropical } \\
\text { úmido }\end{array}$ & $\begin{array}{l}\text { Várzeas litorâneas } \\
\text { e Mata Atlântica }\end{array}$ & $\begin{array}{l}\text { Floresta higrófila } \\
\text { de várzea e } \\
\text { campo higrófilo - } \\
\text { imperfeitamente } \\
\text { drenado, com } \\
\text { drenagem artificial }\end{array}$ & $\begin{array}{l}22^{\circ} 17^{\prime} 13.3^{\prime \prime} \mathrm{S} \\
41^{\circ} 52^{\prime} 15.1^{\prime \prime} \mathrm{W}\end{array}$ & $\begin{array}{l}\text { Organossolo } \\
\text { Háplico sáprico } \\
\text { típico }\end{array}$ & 5 \\
\hline MA05 & $\begin{array}{l}\text { Equatorial } \\
\text { úmido }\end{array}$ & $\begin{array}{l}\text { Matas de galeria, } \\
\text { manguezais e } \\
\text { bacias lacustres }\end{array}$ & $\begin{array}{l}\text { Campo de várzea } \\
\text { higrófilo - } \\
\text { muito mal drenado }\end{array}$ & $\begin{array}{c}3^{\circ} 32^{\prime} 10.0^{\prime \prime} \mathrm{S} \\
45^{\circ} 12^{\prime} 01.3^{\prime \prime} \mathrm{W}\end{array}$ & $\begin{array}{l}\text { Organossolo } \\
\text { Háplico sáprico } \\
\text { típico }\end{array}$ & 5 \\
\hline PR06 & $\begin{array}{l}\text { Subtropical } \\
\text { úmido } \\
\text { mesotérmico }\end{array}$ & $\begin{array}{l}\text { Campos úmidos } \\
\text { e campos gerais }\end{array}$ & $\begin{array}{l}\text { Campo subtropical - } \\
\text { mal drenado }\end{array}$ & $\begin{array}{l}25^{\circ} 14^{\prime} 30.6^{\prime \prime} \mathrm{S} \\
50^{\circ} 01^{\prime} 25.9^{\prime \prime} \mathrm{W}\end{array}$ & $\begin{array}{l}\text { Organossolo } \\
\text { Háplico hêmico } \\
\text { típico }\end{array}$ & 984 \\
\hline PR07 & $\begin{array}{l}\text { Subtropical } \\
\text { úmido } \\
\text { mesotérmico }\end{array}$ & $\begin{array}{l}\text { Campos úmidos } \\
\text { e campos gerais }\end{array}$ & $\begin{array}{l}\text { Campo subtropical - } \\
\text { imperfeitamente } \\
\text { drenado, com } \\
\text { drenagem artificial }\end{array}$ & $\begin{array}{l}25^{\circ} 14^{\prime} 15.4^{\prime \prime} \mathrm{S} \\
50^{\circ} 01^{\prime} 19.7^{\prime \prime} \mathrm{W}\end{array}$ & $\begin{array}{l}\text { Organossolo } \\
\text { Háplico hêmico } \\
\text { típico }\end{array}$ & 976 \\
\hline RJ08 & $\begin{array}{l}\text { Tropical } \\
\text { úmido }\end{array}$ & $\begin{array}{l}\text { Várzeas litorâneas } \\
\text { e Mata Atlântica }\end{array}$ & $\begin{array}{l}\text { Campo higrófilo } \\
\text { de várzea - } \\
\text { muito mal drenado }\end{array}$ & $\begin{array}{l}22^{\circ} 20^{\prime} 24.2^{\prime \prime} \mathrm{S} \\
41^{\circ} 57^{\prime} 19.6^{\prime \prime} \mathrm{W}\end{array}$ & $\begin{array}{l}\text { Organossolo } \\
\text { Háplico hêmico } \\
\text { típico }\end{array}$ & 8 \\
\hline
\end{tabular}

Localização: RJ01 - Silva Jardim, RJ; RJ02 - Silva Jardim, RJ; RJ03 e RJ04 - Macaé, RJ; MA05 - Monção, MA; PR06 - Ponta Grossa, PR; PR07 - Ponta Grossa, PR; e RJ08 - Macaé, RJ.

Os teores de fibras totais (FT) e o de fibras esfregadas (FE) foram determinados segundo Levenque \& Dinel (1977), adaptando o método para lavagem em água corrente sobre peneira de 100 mesh $(0,15 \mathrm{~cm}$ de abertura de malha). $\mathrm{O}$ teor de fibras esfregadas foi determinado, esfregando-se a amostra 10 vezes entre o polegar e $o$ indicador e lavando-a sobre a peneira, lendo o volume de fibras em uma meia seringa cortada longitudinalmente. Os resultados foram expressos em porcentagem de massa, em analogia à análise granulométrica.

O teor de matéria orgânica nos horizontes orgânicos foi determinado pelo método da mufla. Esse é um processo de combustão quantitativo, em que uma massa de solo, previamente seca em estufa $\left(105^{\circ} \mathrm{C}\right.$ por $\left.24 \mathrm{~h}\right)$, foi macerada e peneirada. Depois de pesada, a amostra foi colocada em forno mufla e deixada por $6 \mathrm{~h}$ a $600^{\circ} \mathrm{C}$; decorrido esse tempo, a amostra foi novamente pesada. O teor de matéria orgânica foi estabelecido por diferença de massa em relação à amostra original seca em estufa.

\section{RESULTADOS E DISCUSSÃO}

\section{Ambiente de formação, morfologia e classificação dos Organossolos}

Algumas informações sobre ambiente, posição, altitude e classificação, além da paisagem e do histórico de uso de cada perfil e das características morfológicas dos Organossolos, são reproduzidas do trabalho de Ebeling et al. (2011) e apresentadas nos quadros $1 \mathrm{e}$ 2, complementadas por dados do perfil PR07.

Os perfis RJ01 a RJ04 e o RJ08 (Rio de Janeiro) estão localizados em ambiente tropical úmido, feição de Mata Atlântica em várzeas litorâneas. O perfil MA05 (Maranhão) encontra-se na região préamazônica maranhense, zona equatorial, em feição de várzea e ambiente lagunar. Os perfis PR06 e PR07 (Paraná), na região Sul, estão em ambiente subtropical de planalto e campo subtropical. Nenhum dos perfis apresenta influência marinha atual ou tiomorfismo, 
Quadro 2. Algumas características morfológicas dos Organossolos nos Estados do Rio de Janeiro, Maranhão e Paraná

\begin{tabular}{|c|c|c|c|c|c|}
\hline Perfil & Hor. & Espessura & Cor Munsel (úmida) & Consistência & Estrutura \\
\hline & & $\mathrm{cm}$ & & & \\
\hline \multirow[t]{9}{*}{ RJ01 } & Hd1 & $0-12$ & Preto (10YR 2/1) & muito friável & granular \\
\hline & $\mathrm{Hd} 2$ & $12-24$ & Bruno escuro (10YR 3/3) mesclado & muito friávell & granular \\
\hline & & & Bruno amarelo-escuro (10YR 4/4) & & igeiramente dura \\
\hline & Cg1 & $24-46$ & Cinzento-escuro (5YR 4/1) & firme, ligeiramente dura & maciça \\
\hline & $\mathrm{Cg} 2$ & $46-57$ & Cinzento-rosado (7,5 YR 7/2) & firme, ligeiramente dura & maciça \\
\hline & $2 \mathrm{Hbd} 1$ & $57-76$ & Bruno muito escuro (10YR 2/2) e & & \\
\hline & & & Bruno- avermelhado $(2,5 \mathrm{YR} 4 / 4)$ & macia, muito friável & blocos subangulares \\
\hline & $2 \mathrm{Hbd} 2$ & $76-116$ & Preto (10YR 2/1) & macia, muito friável & maciça \\
\hline & $2 \mathrm{Hbd} 3$ & $116-206^{+}$ & Cinzento muito escuro (10YR 3/2) & macia, firme & maciça \\
\hline \multirow[t]{3}{*}{ RJ02 } & Hdo & 0-19 & Bruno muito escuro (10YR 2/2) & solta, firme & aspecto maciço com poucas fibras \\
\hline & Ho1 & $19-40$ & Cinzento muito escuro (10YR 3/1) & macia, dura & aspecto maciço com muitas fibras \\
\hline & Ho2 & $40-67^{+}$ & Bruno acinzentado muito escuro (10YR 3/2) & muito dura, muito friável & aspecto maciço com muitas fibras \\
\hline \multirow[t]{6}{*}{ RJ03 } & Hdp1 & $0-6$ & Bruno muito escuro (10YR 2/2) & dura, firme & granular e blocos subangulares \\
\hline & Hdp2 & $6-17$ & Cinzento muito escuro (10YR 3/1) & dura, firme & blocos subangulares \\
\hline & $\mathrm{Cg}$ & $17-20$ & Bruno acinzentado (10YR 5/2) & dura, firme & maciça \\
\hline & $2 \mathrm{Hbd}$ & $20-32$ & Preto (10YR 2/1) & dura, firme & maciça \\
\hline & $2 \mathrm{Cbg}$ & $32-38$ & Bruno acinzentado (10YR 5/2) & dura, firme & maciça \\
\hline & $3 \mathrm{Hbd}$ & $38-56^{+}$ & Preto (10YR 2/1) & dura, firme & maciça \\
\hline \multirow[t]{7}{*}{ RJ04 } & Hdp1 & $0-10$ & Preto (10YR 2/1) & dura, friável & moderada pequena granular \\
\hline & Hdp2 & $10-20$ & Preto (10YR 2/1) & muito dura, firme & maciça a fraca pequena granular \\
\hline & $\mathrm{Cg}$ & $20-28$ & Bruno acinzentado-claro (10YR 6/1) & muito dura, firme & maciça \\
\hline & $2 \mathrm{Hbd}$ & $28-33$ & Preto (10YR N 2) & solta, friável & granular \\
\hline & $2 \mathrm{Cbg}$ & $33-38$ & Cinzento-brunado-claro (10YR 6/2) & muito dura, firme & maciça \\
\hline & $3 \mathrm{Hbd} 1$ & $38-53$ & Preto (10YR 2/1) & solta, friável & maciça com fibras \\
\hline & $3 \mathrm{Hbd} 2$ & $53-71^{+}$ & Bruno acinzentado (10YR 5/2) & solta, friável & maciça com fibras \\
\hline \multirow[t]{6}{*}{ MA05 } & Hdo1 & $0-17$ & Preto (10YR N 2) & muito friável, dura & maciça, fraca e \\
\hline & & & & & muito pequena granular \\
\hline & Hdo2 & $17-41$ & Preto (10YR N 2) & muito dura, muito friável & maciça \\
\hline & $\mathrm{Cg}$ & $41-46$ & Bruno muito claro acinzentado (10YR 7/3) & dura, firme & maciça \\
\hline & $2 \mathrm{Hbd} 1$ & $46-61$ & Preto (10YR N 2) & muito dura, muito friável & maciça \\
\hline & $2 \mathrm{Hbd} 2$ & $61-74^{+}$ & Preto (10YR N 2) & muito dura, muito friável & maciça \\
\hline \multirow[t]{5}{*}{ PR06 } & Hdo1 & $0-19$ & Preto (10YR N 2) & macia, muito friável & pequena granular \\
\hline & Hdo2 & $19-42$ & Preto (10YR N 2) & muito friável, & maciça \\
\hline & & & & ligeiramente dura & \\
\hline & Hdo3 & $42-55$ & Preto (10YR 2/1) & friável, ligeiramente dura & maciça \\
\hline & Hdo4 & $55-75^{+}$ & Bruno muito escuro (10 YR 2/2) & dura, friável & maciça \\
\hline \multirow[t]{3}{*}{ PR07 } & Ap & $0-15$ & Bruno muito claro acinzentado(10YR 7/3) & macia, friável & pequena granular \\
\hline & Hdp1 & $15-46$ & Preto $(7,5 Y R$ N2) & muito friável, dura & maciça \\
\hline & Hdp2 & $46-67^{+}$ & Preto $(7,5 Y R$ N2) & dura, friável & maciça \\
\hline \multirow[t]{3}{*}{ RJ08 } & Hdo & $0-18$ & Preto (10YR 2/1) & dura, firme & moderada, pequena e granular \\
\hline & Ho1 & $18-36$ & Bruno muito escuro (10 YR 2/2) & dura, firme & maciça com muitas fibras grandes \\
\hline & Ho2 & $36-58^{+}$ & Bruno muito escuro (10 YR 2/2) & dura, firme & maciça com muitas fibras grandes \\
\hline
\end{tabular}

embora essas condições possam ter ocorrido no passado.

O perfil RJ01, localizado na Reserva Biológica Poço das Antas (REBIO - Casimiro de Abreu/Silva Jardim, RJ) tem menor pressão antrópica; no entanto, a área é periodicamente submetida a queimadas, que duram vários dias. Nessa área, não houve drenagem e não há uso agrícola. O lençol freático encontrava-se a $116 \mathrm{~cm}$ de profundidade. Além da variação de valor e croma nos horizontes, nota-se a estrutura em blocos subangulares no $2 \mathrm{Hbd} 1$, situado abaixo de horizontes $\mathrm{Cg}$ (Quadro 2). O perfil RJ02 localiza-se em Silva
Jardim, em área até pouco tempo com floresta tropical de várzea e recentemente queimada e destocada para implantação de pastagem. Esse perfil apresenta cerca de $5 \%$ em volume de troncos, galhos, arbustos e material (tecido vegetal ainda identificável) apodrecido e uniforme, principalmente nos horizontes Ho1 e Ho2, com muitas fibras. O lençol freático, a $43 \mathrm{~cm}$, coincide com o Ho2.

Os perfis RJ03 e RJ04, em Macaé, RJ, estão em propriedades rurais, com pastagem consorciada em sistema rotacionado e intenso uso agrícola com lavouras anuais rotacionadas de milho e feijão. $\mathrm{Na}$ 
área do perfil RJ03, os drenos têm menor profundidade; no período chuvoso, o lençol está próximo à superfície do solo; no perfil RJ04, a profundidade dos drenos é de 1,5 a 2 m. O lençol freático no perfil RJ03 encontravase a $42 \mathrm{~cm}$ da superfície, enquanto no perfil RJ04, a cerca de $120 \mathrm{~cm}$.

O perfil MA05 foi coletado na área inundável do lago Anananzal, sendo cultivado com lavouras de subsistência de arroz, milho e feijão. O sistema de manejo caracteriza-se por pouca ou nenhuma adoção de práticas de correção da fertilidade do solo, de plantas melhoradas ou controle fitossanitário e, em pequenas áreas, com cultivo manual. O lençol freático estava a $60 \mathrm{~cm}$ da superfície.

Os perfis PR06 e PR07 estão em ambiente de várzea, porém em maior altitude, na região do Planalto em Ponta Grossa, PR. O PR06 teve reduzido uso agrícola e o lençol freático estava a $55 \mathrm{~cm}$ da superfície. O perfil PR07 foi drenado para cultivo de arroz na década de 1980, permanecendo com sistema irrigado por inundação por cerca de 10 anos. Depois, a área foi abandonada, mas ocorreram várias queimadas nos últimos anos. Destaca-se a presença de horizonte Ap (Quadro 2), decorrente da redução do teor de carbono orgânico pela frequência de fogo, indicada pela grande quantidade de cinzas.

Também no Estado do Rio de Janeiro, os perfis RJ08, RJ03 e RJ04 estão em área de várzea, com alagamento sazonal e por longos períodos no ano. $\mathrm{O}$ perfil RJ08 permanece com lençol freático elevado quase todo o ano, sem indícios de alteração pelo uso agrícola. Portanto, foi tomado como referência para comparar variações no estádio de decomposição da matéria orgânica, em razão do uso agrícola e dos efeitos da drenagem sobre a subsidência. O PRJ08 possui muitas fibras grandes, identificáveis visivelmente, além da presença de material lenhoso em estádio intermediário de decomposição. A consistência do material apresentou-se dura quando seco (Quadro 2).

Todos os perfis foram classificados como Organossolos Háplicos (Quadro 1), de acordo com o SiBCS (Santos et al., 2006). O perfil RJ01 foi identificado como Organossolo Háplico hêmico terriço os RJ03, PR06, PR07 e RJ08, como Organossolos Háplicos hêmicos típicos; e os demais, como Organossolos Háplicos sápricos típicos.

\section{Densidade do solo (Ds)}

A Ds variou em razão da proporção dos constituintes das frações orgânicas e minerais do solo e das diversas atividades agrícolas e formas de manejo. Os valores variaram de 0,09 a $0,93 \mathrm{Mg} \mathrm{m}^{-3}$, com média de $0,44 \mathrm{Mg} \mathrm{m}^{-3}$, e desvio-padrão de 0,23 , porém com alto coeficiente de variação (52\%) (Quadro 3).

Valladares et al. (2008), aplicando métodos multicritérios na análise de propriedades de Organossolos, encontraram valores de Ds de 0,07 a
0,80 $\mathrm{Mg} \mathrm{m}^{-3}$, para horizontes orgânicos de diferentes regiões do Brasil. Conceição et al. (1999b) encontraram valores de $\mathrm{Ds}$ em horizontes hísticos variando de 0,05 a $1,06 \mathrm{Mg} \mathrm{m}^{-3}$, com média 0,57 , mediana 0,64 ; a classe modal entre 0,60 e $0,75 \mathrm{Mg} \mathrm{m}^{-3}$, em horizontes orgânicos de solos nas várzeas dos rios Una, Macaé e São João, parte integrante da Baixada Litorânea do Estado do Rio de Janeiro. Kämpf \& Schneider (1989), em seus estudos em solos orgânicos no Rio Grande do Sul, verificaram valores de $\mathrm{Ds}$ variando de $0,07 \mathrm{a}$ $0,38 \mathrm{Mg} \mathrm{m}^{-3}$. Nichols \& Boelter (1984), em turfas de Minnesota, Wisconsin e Michigan encontraram valores de Ds de 0,09 a $0,37 \mathrm{Mg} \mathrm{m}^{-3}$, em que, dentre as 176 amostras avaliadas, $95 \%$ foram inferiores a $0,250 \mathrm{Mg} \mathrm{m}^{-3}$ e menos de $5 \%$, entre 0,250 e $0,374 \mathrm{Mg} \mathrm{m}^{-3}$. Os valores obtidos pelos autores citados estão, em geral, dentro da faixa de variação observada neste estudo (Quadro 3).

As variações nos valores de Ds dos Organossolos estão relacionadas à qualidade da matéria orgânica (espécies vegetais, teor de celulose e lignina), aos componentes da fração mineral (proporção das frações granulométricas areia, silte e argila), ao clima (úmido e, ou, seco), temperatura (alta e, ou, baixa) e umidade (alta e, ou, baixa). Esses fatores interferem no tipo de material orgânico e na interação com o material mineral depositado (sedimentos coluviais e aluviais), nos processos de decomposição (aeróbio e anaeróbio) e nos compostos orgânicos formados (material recalcitrante ou não).

Os valores de Ds apresentaram variações em razão da ocorrência ou não de alterações pelo manejo dos solos para uso agrícola ou pastagens, com ligeiro aumento desse atributo nos horizontes superficiais dos perfis RJ01 (influência de queimadas), RJ03 (revolvimento mecânico), RJ04 (queimada e pisoteio animal), MA05 (deposição sedimentar e variação sasonal) e PR07 (queimada e cultivo), quando comparados com os horizontes subsuperficiais. É diferente do observado nos perfis RJ08 (sem manejo, várzea), PR06 (sem manejo, planalto) e RJ02 (sem manejo, várzea), onde a Ds aumenta em profundidade, em paralelo à diminuição do teor de $\mathrm{MO}$ e ao aumento do teor de MM.

$\mathrm{Na}$ figura 1, é apresentado o diagrama de dispersão e regressão da Ds em razão do teor de carbono ( $r=-0,7443, p<0,001)$, corroborando com dados de Ebeling et al. (2011). Em geral, os Organossolos aumentam a Ds com a drenagem e cultivo. Esses fatores levam ao aumento relativo no teor de MM, com a decomposição da matéria orgânica, e acarretam em maior variação de umidade, portanto, conduzindo à maior agregação do solo. Pode ser observado no quadro 3 que os maiores valores de Ds ocorrem, na maioria dos perfis, em superfície, em horizontes com estrutura do tipo granular (Quadro 2). Segundo Valladares (2003) e Ewing \& Vepraskas (2006), a Ds é facilmente alterada pela subsidência, sendo, portanto, bom indicador de modificação antrópica. 
Quadro 3. Atributos físicos Ds, DMO, RM e MM e teor de matéria orgânica nos horizontes hísticos

\begin{tabular}{|c|c|c|c|c|c|c|c|}
\hline Perfil & Horizonte & Ds & DMO & $\mathbf{R M}$ & RMC & MM & MO \\
\hline & & & 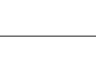 & & & $\%$ & $\mathrm{~g} \mathrm{~kg}^{-1}$ \\
\hline \multirow[t]{5}{*}{ RJ01 } & Hd1 & 0,37 & 0,29 & 0,10 & 0,19 & 53 & 148,80 \\
\hline & $\mathrm{Hd} 2$ & 0,37 & 0,31 & 0,10 & 0,14 & 56 & 116,66 \\
\hline & $2 \mathrm{Hbd} 1$ & 0,38 & 0,28 & 0,12 & 0,22 & 56 & 160,81 \\
\hline & $2 \mathrm{Hbd} 2$ & 0,27 & 0,11 & 0,10 & 0,35 & 53 & 467,39 \\
\hline & $2 \mathrm{Hbd} 3$ & 0,27 & 0,12 & 0,10 & 0,33 & 56 & 443,89 \\
\hline \multirow[t]{3}{*}{ RJ02 } & Hdo & 0,19 & 0,04 & 0,10 & 0,34 & 45 & 745,83 \\
\hline & Ho1 & 0,09 & 0,02 & 0,05 & 0,17 & 36 & 837,16 \\
\hline & Ho2 & 0,09 & 0,03 & 0,04 & 0,14 & 31 & 694,26 \\
\hline \multirow[t]{4}{*}{ RJ03 } & Hdp1 & 0,50 & 0,35 & 0,10 & 0,35 & 68 & 222,68 \\
\hline & Hdp2 & 0,47 & 0,33 & 0,10 & 0,32 & 75 & 254,41 \\
\hline & $2 \mathrm{Hbd}$ & 0,41 & 0,24 & 0,12 & 0,39 & 66 & 335,17 \\
\hline & $3 \mathrm{Hbd}$ & 0,30 & 0,11 & 0,11 & 0,43 & 47 & 530,26 \\
\hline \multirow[t]{5}{*}{ RJ04 } & Hdp1 & 0,48 & 0,33 & 0,10 & 0,33 & 72 & 278,02 \\
\hline & Hdp2 & 0,53 & 0,36 & 0,11 & 0,39 & 61 & 392,33 \\
\hline & $2 \mathrm{Hbd}$ & 0,44 & 0,27 & 0,11 & 0,38 & 64 & 362,07 \\
\hline & $3 \mathrm{Hbd} 1$ & 0,30 & 0,18 & 0,08 & 0,27 & 65 & 348,10 \\
\hline & $3 \mathrm{Hbd} 2$ & 0,30 & 0,15 & 0,10 & 0,34 & 58 & 415,75 \\
\hline \multirow[t]{4}{*}{ MA05 } & Hdo1 & 0,61 & 0,40 & 0,14 & 0,47 & 64 & 355,48 \\
\hline & Hdo2 & 0,53 & 0,40 & 0,12 & 0,29 & 79 & 208,25 \\
\hline & $2 \mathrm{Hbd} 1$ & 0,49 & 0,43 & 0,09 & 0,14 & 67 & 131,00 \\
\hline & $2 \mathrm{Hbd} 2$ & 0,49 & 0,43 & 0,09 & 0,13 & 66 & 143,11 \\
\hline \multirow[t]{4}{*}{ PR06 } & Hdo1 & 0,63 & 0,33 & 0,14 & 0,68 & 59 & 405,06 \\
\hline & Hdo2 & 0,72 & 0,41 & 0,16 & 0,71 & 71 & 291,51 \\
\hline & Hdo3 & 0,93 & 0,62 & 0,21 & 0,71 & 81 & 154,17 \\
\hline & Hdo4 & 0,93 & 0,66 & 0,18 & 0,61 & 87 & 126,67 \\
\hline \multirow[t]{2}{*}{ PR07 } & Hdp1 & 0,85 & 0,49 & 0,16 & 0,81 & 83 & 372,33 \\
\hline & Hdp2 & 0,79 & 0,51 & 0,19 & 0,64 & 85 & 148,95 \\
\hline \multirow[t]{3}{*}{ RJ08 } & Hdo & 0,12 & 0,05 & 0,05 & 0,18 & 44 & 556,92 \\
\hline & Ho1 & 0,21 & 0,04 & 0,11 & 0,37 & 43 & 773,99 \\
\hline & Ho2 & 0,23 & 0,05 & 0,09 & 0,41 & 49 & 713,81 \\
\hline Máximo & & 0,93 & 0,66 & 0,21 & 0,81 & 87 & 837,16 \\
\hline Mínimo & & 0,09 & 0,02 & 0,04 & 0,13 & 31 & 116,66 \\
\hline Médio & & 0,44 & 0,28 & 0,11 & 0,37 & 61 & 371,16 \\
\hline $\mathrm{Dp}$ & & 0,23 & 0,18 & 0,04 & 0,19 & 14,41 & 213,62 \\
\hline CV (\%) & & 52,06 & 63,68 & 34,01 & 50,59 & 23,49 & 57,55 \\
\hline
\end{tabular}

Ds: Densidade do solo; DMO: Densidade da matéria orgânica; RM: Resíduo mínimo; RMC: Resíduo mínimo com base na média da Ds desses solos; MM: Material mineral; Dp: Desvio-padrão; CV: Coeficiente de variação; e MO: matéria orgânica determinada em forno mufla.

Porém, a determinação da Ds apresenta algumas limitações, em especial a variabilidade do material orgânico, umidade do solo, alteração de volume com a contração e expansão e estágio de decomposição da MOS.

\section{Densidade da matéria orgânica (DMO)}

Os valores calculados de DMO variaram de 0,02 a $0,66 \mathrm{Mg} \mathrm{m}^{-3}$, sendo a média de $0,28 \mathrm{Mg} \mathrm{m}^{-3}$ (Quadro 3). Conceição et al. (1999b), estudando Organossolos no Estado do Rio de Janeiro, encontraram valores nessa ordem, sendo o menor de $0,07 \mathrm{Mg} \mathrm{m}^{-3}$. Valores dessa magnitude também foram verificados por Lynn et al. (1974). Os autores relataram que valores de DMO acima de $0,07 \mathrm{Mg} \mathrm{m}^{-3}$ corresponderam a solos cultivados. Os perfis RJ02 e RJ08 apresentam valores de DMO inferiores a $0,07 \mathrm{Mg} \mathrm{m}^{-3}$, corroborando com o exposto por Lynn et al. (1974). Esses perfis foram usados como referência, pois não têm uso agrícola, estão saturados por água boa parte do ano e não apresentam nenhuma influência 
antrópica. Em estudo com Organossolos de várias regiões do Brasil, Valladares (2003) encontrou valores de DMO de 0,01 a 0,32 $\mathrm{Mg} \mathrm{m}^{-3}$, porém, não observou diferenças significativas nos valores de $\mathrm{DMO}$ em razão do ambiente.

Para os perfis RJ01, RJ03 e RJ04, foi observada redução da $\mathrm{DMO}$ em profundidade, indicando o maior grau de humificação e mineralização da matéria orgânica nas camadas superficiais. No perfil PR06, localizado em clima mais frio, a DMO aumentou em profundidade, observando-se correlação altamente significativa $(r=0,95, p<0,01)$ entre Ds e DMO (Figura 2a).

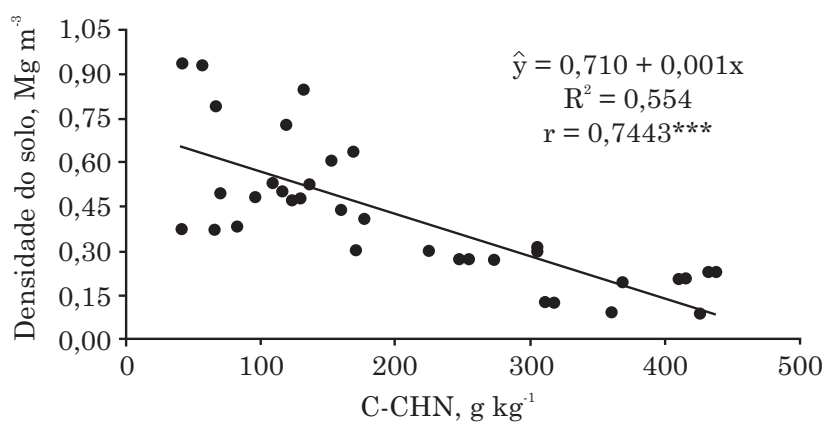

Figura 1. Diagrama de dispersão e regressão da densidade do solo em função do teor de carbono (C determinado pelo método de combustão a seco em analisador elementar CHN). Fonte: Ebeling et al. (2011).
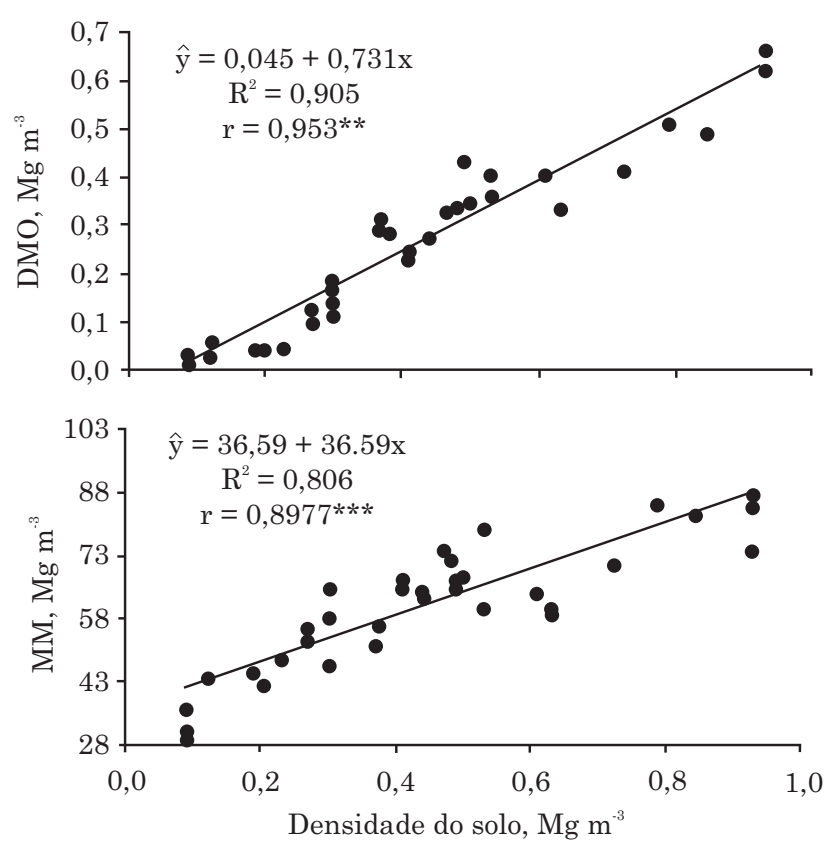

Figura 2. Correlação entre (a) densidade do solo (Ds) e densidade da matéria orgânica (DMO) e (b) densidade do solo (Ds) e \% de material mineral (\%MM).

\section{Porcentagem de material mineral (MM)}

O MM variou aproximadamente de 30 a $87 \%$, com valores médios de $61 \%$, coeficiente de variação de $23 \%$ e desvio-padrão de 14 (Quadro 3). Em Organossolos no Estado do Rio de Janeiro, Conceição et al. (1999b) encontraram variação de MM de 28 a $83 \%$. Estudando Histosols sob floresta no sudoeste do Alasca, D’More \& Lynn (2002) encontraram variação de MM desde 1 a $93 \%$.

O coeficiente de correlação entre Ds e MM (Figura $2 \mathrm{~b}$ ) indicou proporcionalidade direta entre os atributos $(\mathrm{r}=0,90, \mathrm{p}<0,001)$. Os valores mais elevados de MM devem-se ao intenso uso das áreas, acelerando a decomposição do material orgânico com aumento relativo da fração mineral do solo. Os perfis PR06 e PR07 apresentaram os maiores valores de material mineral. Mantida a condição atual de uso e drenagem, esses perfis terão, provavelmente, baixo potencial de transformação, apresentando, além do elevado teor de $\mathrm{MM}$, maior Ds $\left(0,72\right.$ a $\left.0,92 \mathrm{Mg} \mathrm{m}^{-3}\right)$ e maior $\mathrm{RM}(0,14$ a $0,21 \mathrm{~cm} \mathrm{~cm}^{-1}$ ).

\section{Resíduo mínimo (RM)}

Na figura 3, é apresentado o diagrama de dispersão do $\mathrm{RM}$ em razão da Ds, com correlação positiva $(r=0,90, p<0,001)$ entre as variáveis. Esses atributos estão relacionados ao potencial de subsidência e quanto maiores os valores de Ds e RM menor a intensidade desse processo. Nos perfis estudados, o RM variou de $0,04 \mathrm{~cm} \mathrm{~cm}^{-1}$ a $0,21 \mathrm{~cm} \mathrm{~cm}^{-1}$, com média de $0,11 \mathrm{~cm} \mathrm{~cm}^{-1}$ (Quadro 3). Os maiores valores de RM foram observados nos perfis PR06 e PR07, em várzea de maior altitude e relativamente com menor hidromorfismo ao longo do ano. A maior oxidação da matéria orgânica foi favorecida pela drenagem na década de 1980 (perfil PR07), acentuando a mineralização mesmo nos horizontes subsuperficiais.

Valladares (2003) encontrou valores de RM entre 0,003 e $0,580 \mathrm{~cm} \mathrm{~cm}^{-1}$, com média de $0,17 \mathrm{~cm} \mathrm{~cm}^{-1}$, evidenciando que amostras com RM inferior a $0,01 \mathrm{~cm} \mathrm{~cm}^{-1}$ apresentaram teores muito elevados de MO e baixa Ds. Portanto, essa propriedade pode ser usada para definição de áreas de Organossolos

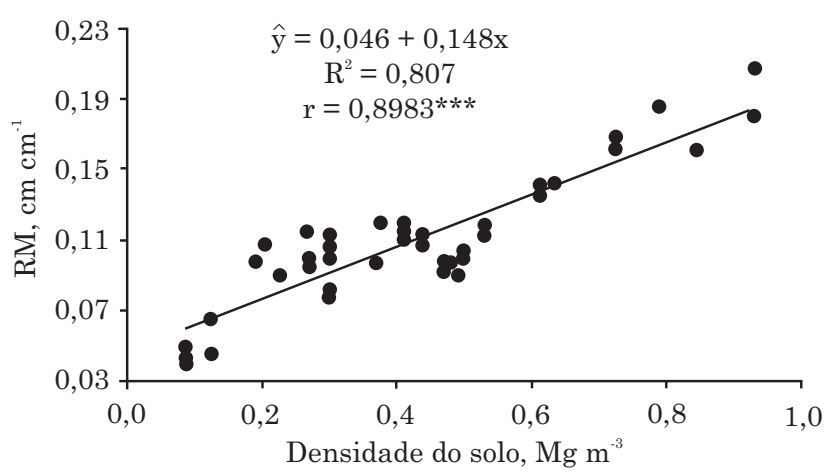

Figura 3. Correlação entre a densidade do solo (Ds) e o resíduo mínimo (RM). 
destinadas à preservação ambiental, como reservatórios e filtragem e armazenamento de água, por indicar a maior suscetibilidade à degradação pela subsidência.

Para o cálculo do resíduo mínimo, foi também usado o valor médio da Ds das amostras dos Organossolos (média $=0,44 \mathrm{Mg} \mathrm{m}^{-3}$ ), sendo identificado como RMC (Quadro 3), segundo proposto por Valladares (2003), em vez do valor de $1,5 \mathrm{Mg} \mathrm{m}^{-3}$ arbitrado por Lynn et al. (1974). Os valores de RMC são mais altos que os de RM, com máximo de $0,81 \mathrm{~cm} \mathrm{~cm}^{-1}$, média de $0,37 \mathrm{~cm} \mathrm{~cm}^{-1}$ e mínimo de $0,13 \mathrm{~cm} \mathrm{~cm}^{-1}$. $\mathrm{Na}$ figura 4, observa-se o diagrama de dispersão do RM em razão do RMC, em que a distribuição dos pontos indica grande variação dos dados, que pode ser por causa dos métodos de determinação do RM e da Ds em Organossolos.

Os perfis localizados no Paraná apresentaram os maiores valores de RM (Quadro 3) e os menores foram verificados para os perfis RJ02, seguidos do RJ08 e MA05, indicando para esses solos elevados risco de degradação. Os perfis RJ03 e RJ04 evidenciaram risco moderado de degradação; os PR06 e PR07, nas condições atuais de uso e drenagem, menor risco.

\section{Grau de Decomposição - Von Post, índice de pirofosfato e teor de fibras}

Aplicando a classificação de Von Post (1922), dos 30 horizontes hísticos, nove foram classificados como sápricos; 20, como hêmicos; e um, como fíbrico (Quadro 4). O ambiente tropical favorece a transformação da MOS e mineralização e, ou, humificação, diminuindo os teores de fibras e levando ao predomínio de materiais sápricos e hêmicos. Nos horizontes hísticos superficiais dos perfis estudados, embora sendo marcante a transformação do material orgânico, ainda predominam características hêmicas. Já os subsuperficiais são em geral sápricos, possivelmente refletindo a alternância de condições de deposição de sedimentos, redução e mineralização, em várias fases de pedogênese, indicadas pela alternância de horizontes hísticos e minerais $(\mathrm{Cg})$. Em ambientes onde o revolvimento do solo é mais intenso, com operações de gradagem e incorporação

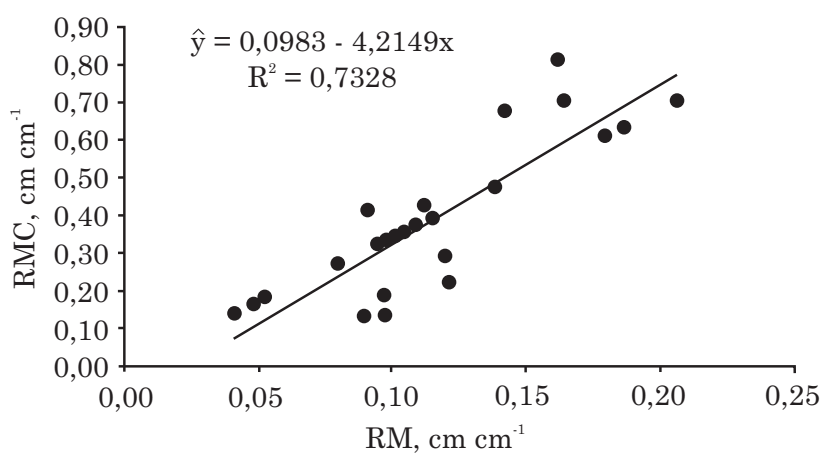

Figura 4. Dispersão entre as duas formas de cálculo do RM, variando o valor de Ds utilizado pela média de Ds dos solos e usando o valor de $1,5 \mathrm{Mg} \mathrm{m}^{-3}$. de adubos com frequência anual, a subsidência é ainda mais acentuada, como visto no perfil RJ03, hêmico, em comparação ao perfil RJ04, sáprico. As duas áreas são próximas geograficamente e a variação nas propriedades do material orgânico deveu-se ao nível do lençol freático. A drenagem nos perfis RJ03 e RJ04, para produção de culturas anuais, acelerou a subsidência e intensificou a mineralização da MOS.

Quanto ao grau de decomposição do material orgânico, avaliado pelo índice em pirofosfato, três amostras foram classificadas como de material fíbrico (Hd2 - RJ01, Ho1 e Ho2 - RJ08), seis como sáprico e 21, como de material hêmico (Quadro 4).

Para a avaliação de Organossolos quanto ao grau de degradação, as variáveis índice de pirofosfato e teor de fibras esfregadas foram usadas em conjunto (Figura 5), o que facilitou a classificação dos materiais orgânicos, pois as classes são delimitadas por uma faixa de valores. O Sistema Brasileiro de Classificação de Solos - SiBCS (Santos et al., 2006) adota limites rígidos na identificação dos materiais fibricos, hêmicos ou sápricos. Entretanto, os resultados sugerem que são importantes para essa classificação, além dos teores de fibras esfregadas, associações com outros índices, como o índice de pirofosfato (Lynn et al., 1974).

Os teores de fibras esfregadas nas amostras variaram entre 4 e $16 \%$, indicando estádio avançado de decomposição para a maioria dos horizontes. $\mathrm{Na}$ figura 6, é apresentada a relação dos teores de fibras esfregadas com o MM. Porém, a classificação do tipo de material orgânico pode não ser precisa, pois está baseada no teor de fibras e em quantidade estimada de MM. O predomínio de materiais com baixos teores de fibras esfregadas nos Organossolos estudados reflete fatores como clima, uso da terra e tipo de vegetação que deu origem aos materiais orgânicos do solo. Muitos dos horizontes dos perfis avaliados apresentavam uso agrícola e drenagem artificial, o que favoreceu a aeração das camadas superficiais e redução dos teores de fibras. Os perfis RJ01, RJ02 e RJ08 foram os que apresentaram os maiores teores de fibras (Quadro 4), pois são os que têm menor influência antrópica.

\section{Matéria orgânica do solo (MOS)}

Quanto ao teor de matéria orgânica (Quadro 3), o valor médio foi de $371,2 \mathrm{~g} \mathrm{~kg}^{-1}$, com o máximo de $837,2 \mathrm{~g} \mathrm{~kg}^{-1}$ (horizonte Ho1, RJ02) e o mínimo de $116,7 \mathrm{~g} \mathrm{~kg}^{-1}$ (horizonte Hd2, RJ01), com coeficiente de variação de $57,5 \%$. Os teores de MOS foram maiores nos perfis RJ02 e RJ08, ambos com reduzida influência antrópica e com grande quantidade de fibras, onde os atributos Ds, DMO e MM apresentaram os menores valores.

Os perfis, que apresentaram marcas de revolvimento do solo e, ou, influência de queimadas, foram avaliados individualmente quanto às variações 
Quadro 4. Classificação do grau de decomposição do material de solo, segundo a escala de Von Post, o índice de pirofosfato (IP) e o teor de fibras e fibras esfregadas

\begin{tabular}{|c|c|c|c|c|c|}
\hline Amostra V & Von Post & Material & IP & Fibras & $\begin{array}{c}\text { Fibras } \\
\text { esfregadas }\end{array}$ \\
\hline & & & & & $\%$ \\
\hline RJ01-Hd1 & H3 & Fíbrico & 4 & 16 & 8 \\
\hline RJ01-Hd2 & $\mathrm{H} 4$ & Hêmico & 6 & 16 & 4 \\
\hline RJ01-Cg1 & - & Não se aplica & - & & \\
\hline RJ01-Cg2 & - & Não se aplica & - & & \\
\hline RJ01-2Hbd1 & $\mathrm{H} 6$ & Hêmico & 4 & 8 & 4 \\
\hline RJ01-2Hbd2 & H5 & Hêmico & 3 & 16 & 4 \\
\hline RJ01-2Hbd3 & $\mathrm{H} 7$ & Sáprico & 3 & 28 & 4 \\
\hline RJ02-Hdo & H5 & Hêmico & 3 & 40 & 8 \\
\hline RJ02-Ho1 & H7 & Sáprico & 4 & 40 & 8 \\
\hline RJ02-Ho & $\mathrm{H} 7$ & Sáprico & 4 & 40 & 8 \\
\hline RJ03-Hdp1 & H5 & Hêmico & 4 & 12 & 4 \\
\hline RJ03-Hdp2 & H5 & Hêmico & 4 & 8 & 4 \\
\hline RJ03-Cg & - & Não se aplica & - & & \\
\hline RJ03-2Hbd & H5 & Hêmico & 4 & 8 & 4 \\
\hline RJ03-Cbg & - & Não se aplica & - & & \\
\hline RJ03-3Hbd & $\mathrm{H} 6$ & Hêmico & 3 & 8 & 4 \\
\hline RJ04-Hdp1 & $\mathrm{H} 6$ & Hêmico & 5 & 8 & 4 \\
\hline RJ04-Hdp2 & H6 & Hêmico & 5 & 8 & 4 \\
\hline RJ04-Cg & - & Não se aplica & - & & \\
\hline RJ04-2Hbd & $\mathrm{H} 7$ & Sáprico & 4 & 8 & 4 \\
\hline RJ04-Cbg & - & Não se aplica & - & & \\
\hline RJ04-3Hbd1 & $\mathrm{H} 7$ & Sáprico & 4 & 8 & 4 \\
\hline RJ04-3Hbd2 & $\mathrm{H} 7$ & Sáprico & 3 & 8 & 4 \\
\hline MA05-Hdo1 & H8 & Sáprico & 5 & 12 & 4 \\
\hline MA05-Hdo2 & $\mathrm{H} 6$ & Hêmico & 4 & 8 & 4 \\
\hline MA05-Cg & - & Não se aplica & - & & \\
\hline MA05-2Hbd1 & H8 & Sáprico & 4 & 8 & 4 \\
\hline MA05-2Hbd2 & $\mathrm{H} 8$ & Sáprico & 5 & 8 & 4 \\
\hline PR06-Hdo1 & H5 & Hêmico & 4 & 8 & 4 \\
\hline PR06-Hdo2 & H5 & Hêmico & 4 & 8 & 4 \\
\hline PR06-Hdo3 & $\mathrm{H} 6$ & Hêmico & 4 & 8 & 4 \\
\hline PR06-Hdo4 & $\mathrm{H} 6$ & Hêmico & 4 & 8 & 4 \\
\hline PR07-Ap & - & - & - & & \\
\hline PR07-Hdp1 & H5 & Hêmico & 4 & 20 & 4 \\
\hline PR07-Hdp2 & H5 & Hêmico & 4 & 8 & 4 \\
\hline RJ08-Hdo & $\mathrm{H} 6$ & Hêmico & 3 & 8 & 4 \\
\hline RJ08-Ho1 & H5 & Hêmico & 6 & 40 & 8 \\
\hline RJ08-Ho2 & $\mathrm{H} 5$ & Hêmico & 6 & 40 & 16 \\
\hline
\end{tabular}

nos teores de MOS. No perfil RJ01, na Reserva Biológica Poço das Antas, verificou-se variação do horizonte superficial $\mathrm{Hd} 1$ para o $2 \mathrm{Hbd} 3$, a mais profundo, de $33,5 \%$. Na área do perfil RJ03, atualmente com pastagem e drenagem superficial, foi observada redução de $42,0 \%$ entre os horizontes Hdp1 e 3Hbd. Já no perfil RJ04, com cultivo de lavouras

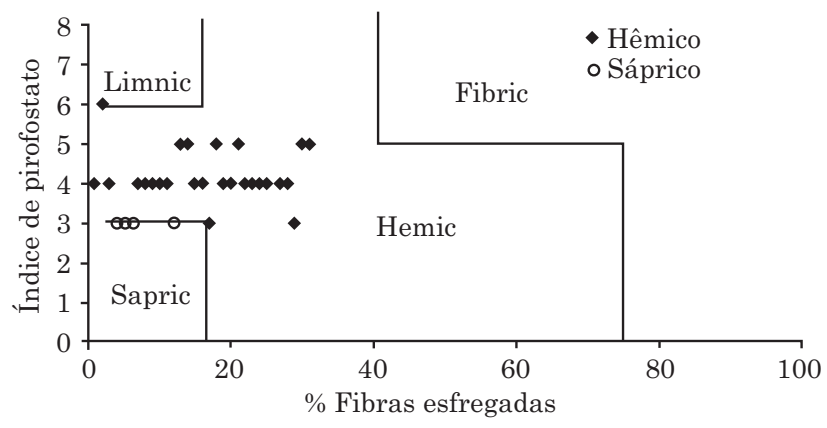

Figura 5. Classificação do material orgânico, segundo a relação entre método químico (índice de pirofosfato) e método físico (teor de fibras esfregadas).

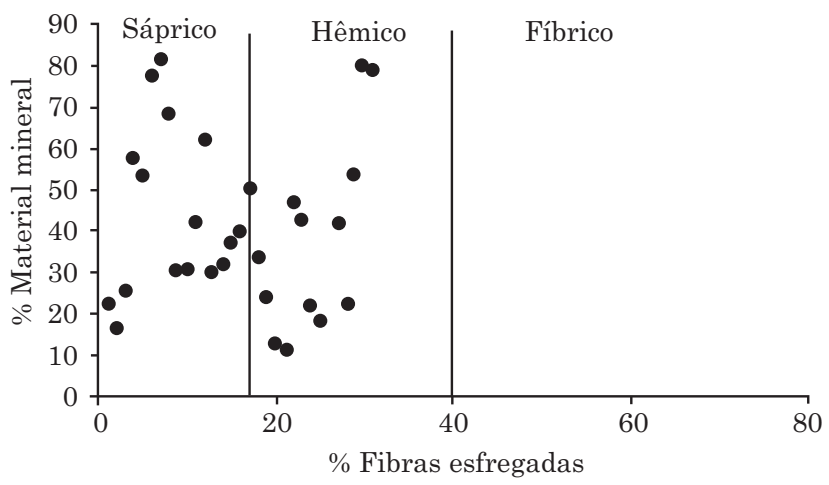

Figura 6. Relação entre os teores de fibras esfregadas $(\% \mathrm{v} / \mathrm{v})$ e o teor de material mineral (\%p/p).

anuais e drenagem profunda, o decréscimo na MOS no horizonte superficial foi de $66,9 \%$. Os perfis RJ03 e RJ04 também apresentaram maiores valores dos atributos DMO e MM no horizonte superficial, diminuindo em profundidade.

Os perfis PR06 e PR07, em ambiente de planícies interioranas, clima subtropical Cfb e com precipitações bem distribuídas, apresentaram predominância de material hêmico; relação $\mathrm{C} / \mathrm{N}$ (carbono/nitrogênio) entre 14 e 20 ; Ds entre 0,63 e $0,93 \mathrm{Mg} \mathrm{m}^{-3}$; RMC de 0,61 a $0,81 \mathrm{Mg} \mathrm{m}^{-3}$; e MM entre 59 e $87 \%$. Indicando a presença de material orgânico mais resistente, esses Organossolos podem ser menos suscetíveis a alterações pelo processo de subsidência, se utilizados para agricultura. $\mathrm{O}$ mesmo pode ser observado para o perfil MA05, em ambiente de clima tropical Awh, o que é explicado pela frequente deposição de novos materiais oriundos de outras partes da paisagem e, muitas vezes, de compostos mais resistentes (aromáticos funcionalizados e polimetilenos).

Os perfis RJ03 e RJ04, de ambiente tropical atlântico Aw, apresentaram Ds entre 0,30 e $0,53 \mathrm{Mg} \mathrm{m}^{-3}, \mathrm{RMC}$ de 0,27 a $0,39 \mathrm{Mg} \mathrm{m}^{-3}$ e MM entre 46 e $72 \%$; o material é menos recalcitrante que o dos perfis anteriores; a suscetibilidade deles à subsidência é moderada; e o cultivo agrícola pode conduzir à degradação seletiva 
da matéria orgânica mais lábil e com isso ao acúmulo relativo de material mais recalcitrante em superfície.

Os perfis RJ01, RJ02 e RJ04, de ambiente tropical atlântico Aw, apresentaram Ds entre 0,09 e $0,38 \mathrm{Mg} \mathrm{m}^{-3}, \mathrm{RMC}$ de 0,14 a $0,41 \mathrm{Mg} \mathrm{m}^{-3}, \mathrm{MM}$ entre 30 e $56 \%$. A presença de materiais com maiores teores de carbono lábil e valores superiores a $40 \%$ demonstra que esses solos exibem alto potencial de perdas por oxidação e mineralização da MOS. O perfil RJ08 apresentou-se como o de maior potencial de subsidência e degradação, tendo a menor quantidade de material mais recalcitrante.

\section{CONCLUSÕES}

1. As propriedades físicas avaliadas: densidade do solo, densidade da matéria orgânica, material mineral e resíduo mínimo refletiram as modificações no teor e grau de decomposição do material orgânico encontrado, nos distintos ambientes e nas condições de uso agrícola dos Organossolos.

2. O teor e o grau de decomposição do material orgânico foram influenciados pelo ambiente de formação, clima, relevo e uso do solo, principalmente a drenagem e o cultivo agrícola. As características do material orgânico depositado, durante a formação dos horizontes em diferentes períodos, influenciaram a labilidade e resistência dos compostos orgânicos formados, contribuindo para a maior ou menor resistência à decomposição.

\section{AGRADECIMENTOS}

Ao Conselho Nacional de Desenvolvimento Científico e Tecnológico - CNPq, pela bolsa e pelo apoio financeiro para execução da tese de Doutorado. Ao CPGA-CSUFRRJ e à Embrapa Solos, pelo suporte de laboratório.

\section{LITERATURA CITADA}

CARTER, R.M. Soil sampling and methods of analysis. Ann Arbor, Lewis Publishers,1993. p.423-471.

CONCEIÇÃO, M.; MANZATTO, C.V.; ARAÚJO, W.S.; MARTIN NETO, L.; SAAB, S.C.; CUNHA, T.J.F. \& FREIXO, A.A. Caracterização de solos orgânicos do Estado do Rio de Janeiro, propriedades físicas e morfológicas como subsídios à classificação. Rio de Janeiro, Embrapa Solos, 1999. 6p. (Pesquisa em Andamento, 4)

D'MORE, D.V. \& LYNN, W.C. Classification of forested Histosols in Southeast Alaska. Soil Sci. Soc. Am. J., 66:554$562,2002$.
EBELING, A.G.; ANJOS, L.H.C.; PEREZ, D.V.; PEREIRA, M.G. \& GOMES, F.W.F. Atributos químicos, carbono orgânico e substâncias húmicas em Organossolos Háplicos de várias regiões do Brasil. R. Bras. Ci. Solo, 35:325-336, 2011.

ELDER, J.W. \& LAL, R. Tillage effects on physical properties of agricultural organic soils of north central Ohio. Soil Till. Res., 98:208-210, 2008.

EWING, J.M. \& VEPRASKAS, M.J. Estimating primary and secondary subsidence in an organic soil 15, 20, and 30 years after drainage. Wetlands, 26:119-130, 2006 .

GALVÃO, F.A.D. \& VAHL, L.C. Calibração do método SMP para solos orgânicos. R. Bras. Agroci., 2:121-131, 1996.

GESCH, R.W.; REICOSKYA, D.C.; GILBERT, R.A. \& MORRIS, D.R. Influence of tillage and plant residue management on respiration of a Florida Everglades Histosol. Soil Till. Res., 92:156-166, 2007.

KÄMPF, N. \& SCHNEIDER, P. Caracterização de solos orgânicos do Rio Grande do Sul: Propriedades morfológicas e físicas como subsídios à classificação. R. Bras. Ci. Solo, 13:227-236, 1989.

LEPSCH, I.F.; QUAGGIO, J.A.; SAKAI, E.; CAMARGO, O.A. \& VALADARES, J.M.A.S. Caracterização, classificação e manejo agrícola de solos orgânicos do vale do Rio Ribeira de Iguape, SP. Campinas, Instituto Agronômico de Campinas, 1990. 58p. (Boletim Técnico, 131)

LEVENQUE, M. \& DINEL, H. Fiber content, particle-size distribution and some related properties of four peat materials in Eastern Canada. Can. J. Soil Sci., 57:187195, 1977.

LÜTZOW, M.V.; KÖGEL-KNABNER, I.; EKSCHMITT, K.; MATZNER, E.; GUGGENBERGER, G.; MARSCHNER, B.\& FLESSA, H. Stabilization of organic matter in temperate soils: Mechanisms and their relevance under different soil conditions - a review. Eur. J. Soil Sci., 57:426-445, 2006.

LYNN, W.C.; MC KINZIE, W.E. \& GROSSMAN, R.B. Field laboratory tests for characterization of Histosols. In: AANDAHL, A.R.; BUOL, S.W.; HILL, D.E.\& BAILEY, H.H., eds. Histosols their characteristics, classification, and use. Madison, SSSA, 1974. p.11-20. (Special Publication, 6)

NICHOLS, D.S. \& BOELTER, D.H. Fiber size distribution, bulk density, and ash content of peats in Minnesota, Wisconsin, and Michigan. Soil Sci. Soc. Am. J., 48:13201328, 1984.

PUMPANEN, J.; KOLARI, P.; ILVESNIEMI, H.; MINKKINEN, K.; VESALA, T.; NIINISTÖ, S.; LOHILA, A.; LARMOLA, T.; MORERO, M.; PIHLATIE, M.; JANSSENS, I.; YUSTE, J.C.; GRÜNZWEIG, J.M.; RETH, S.; SUBKE, J.A.; SAVAGE, K.; KUTSCH, W.; ØSTRENG, G.; ZIEGLER, W.; ANTHONI, P.; LINDROTH, A. \& HARI, P. Comparison of different chamber techniques for measuring soil $\mathrm{CO}_{2}$ efflux. Agric. For. Meteorol., 123:159-176, 2004. 
SAKAI, E. \& LEPSCH, I.F. Levantamento pedológico detalhado e distribuição espacial de características dos solos do Pôlder Registro-I, SP. Campinas, Instituto Agronômico de Campinas, 1987. 56p. (Boletim Científico, 10)

SANTOS, G.S.; JACOMINE, P.K.T.; ANJOS, L.H.C.; OLIVEIRA, V.A.; OLIVEIRA, J.B.; COELHO, M.R.; LUMBRELAS, J.F. \& CUNHA, T.J.F. Sistema brasileiro de classificação de solos. 2.ed. Brasília, Embrapa Produção da Informação; Rio de Janeiro, Embrapa Solos, 2006. 306p.

SANTOS, R.D.; LEMOS, R.C.; SANTOS, H.G.; KER, J.C. \& ANJOS, L.H.C. Manual de descrição e coleta de solo no campo. 5.ed. Viçosa, MG, Sociedade Brasileira de Ciência do Solo, 2005. 92p.
SILVA, A.C.; HORÁK, I.; TORRADO, P.C.; CORTIZAS, A.M.; RACEDO, J.R. \& CAMPOS, J.R.R. Turfeiras da serra do espinhaço meridional - MG. II - Influência da drenagem na composição elementar e substâncias húmicas. R. Bras. Ci. Solo, 33:1399-1408, 2009.

VALLADARES, G.S. Caracterização de Organossolos, auxílio à sua classificação. Seropédica, Universidade Federal Rural do Rio de Janeiro, 2003.142p. (Tese de Doutorado)

VALLADARES, G.S.; PEREIRA, M.G.; ANJOS, L.H.C. \& EBELING, A.G. Caracterização de solos brasileiros com elevados teores de material orgânico. Magistra, 20:95$104,2008$.

VON POST, L. Sveriges geologiska undersöknings torvinventering och nåogra av dess hittils vaanna resultat, (In Swedish ) Sven. Mosskulturfören. Tidskr, 33:1-27, 1922. 